\title{
Aplicación de tecnología cloud computing en bibliotecas y centros de documentación
}

\author{
Application of cloud computing technology in libraries and documentation centres:
} the examples of OCLC's WorldShare and CONTENTdm

\author{
Francisco José VALENTín RUIZ, Robert TORRE-MARÍN AMORós \\ Doc6, España \\ \{vruiz|rtorremarin\}@doc6.es
}

\begin{abstract}
Resumen
La evolución de la automatización de bibliotecas establecida en cuatro periodos - pre-MARC, después de OCLC, después de Internet y socialización de Internet- permite explicar la aparición del cloud computing aplicado a bibliotecas y centros de documentación como un servicio alternativo a los tradicionales sistemas integrados de gestión de bibliotecas (SIGB). En base a esta evolución, se analizan las ventajas e inconvenientes de los servicios en la nube. Estas características se ponen en relación con dos de los servicios de OCLC: WorldShare para bibliotecas y CONTENTdm de gestión de contenidos digitales. De estos dos servicios se ofrecen ejemplos de uso.
\end{abstract}

Palabras clave: Automatización de bibliotecas. Cloud computing. Servicios en la nube. OCLC. WorldShare. CONTENTdm.

\section{Introducción}

La comprensión de cómo el uso de los modelos en la nube o la tecnología cloud computing está extendiéndose en las bibliotecas y centros de documentación requiere del análisis de la evolución histórica de la automatización de bibliotecas. Mostrar esa evolución es uno de nuestros objetivos, aunque el principal es definir las características de estos modelos, sus ventajas e inconvenientes. A partir de estas características pretendemos describir dos modelos de aplicación desarrollados por OCLC: WorldShare para la gestión de bibliotecas y CONTENTdm para la administración y difusión de contenidos digitales, y mostrar cómo satisfacen los requisitos establecidos.

\section{Hacia el modelo cloud computing}

Podemos definir la automatización de bibliotecas y centros de documentación como el procesamiento automático y semiautomático de datos para desarrollar las actividades que tradicionalmente desempeñaban estos centros mediante métodos manuales (Salmon, 1975, p. 338; García Camarero y García Melero, 2001, p. 21). Actualmente es difícil interpretar a qué nos refe-

\begin{abstract}
The evolution of library automation established in four periods -pre-MARC, after of OCLC, after Internet and Internet socialization- can explain the birth of cloud computing applied to libraries and documentation centres as an alternative to traditional integrated library systems (ILS). Based on this technological development, the advantages and disadvantages of cloud services are analyzed. In this work, these features are linked with two OCLC services: WorldShare for libraries and CONTENTdm a digital collection management software. Of these two services examples of use are showed.
\end{abstract}

Keywords: Library automation. Cloud computing. OCLC. WorldShare. CONTENTdm.

rimos cuando hacemos mención a estos métodos manuales y por este motivo hemos de analizar con más detenimiento la evolución de la automatización. Rayward (2002, p.13) distingue tres periodos en esta evolución: pre-MARC; después de OCLC (cuya fundación es paralela a la aparición del formato MARC); y después de Internet. A estas tres etapas debemos añadir una más denominada «socialización de Internet» (Valentín Ruiz; Fernández Sánchez; TorreMarín, 2012, p. 3), en la que tienen cabida las últimas tendencias tecnológicas en torno a las funcionalidades relacionadas con la Web 2.0, al software libre y a las que tienen que ver con el nacimiento de las aplicaciones basadas en la nube (cloud computing) que trataremos de explicar más profusamente.

La etapa pre-MARC podemos establecerla desde que aparecieron los primeros sistemas de automatización a finales de los años 30 del siglo $X X$, a partir de computadoras en las que los datos se introducían mediante tarjetas perforadas (Salmon, 1975, p. 338), hasta que se desarrolló el formato MARC, que estableció un estándar válido para el intercambio de datos entre sistemas y que inició su andadura en 1968 (Reynolds, 1993, p. 473). Los primeros sistemas 
se basaban en el traslado prácticamente directo de los catálogos manuales de las bibliotecas (compuestos por un mueble lleno de fichas con referencias a los distintos materiales de la biblioteca) a una estructura informática más o menos sencilla. Los servicios bibliotecarios que implicaban tradicionalmente mayores necesidades de recursos, como la circulación, también fueron los primeros en informatizarse.

En cuanto a OCLC, cuyas siglas corresponden actualmente a «Online Computer Library Center» pero que originalmente atendieron al lugar de su fundación "Ohio College Library Center», podemos afirmar que supuso todo un hito en la automatización de bibliotecas al fundar el primer sistema de catalogación colaborativa en línea empleando la estructura MARC en 1971 (OCLC, 2012). Desde entonces OCLC ha marcado el ritmo de muchos de los proyectos de cooperación entre bibliotecas y centros de documentación de todo tipo, e incluso ha impuesto la tendencia de algunos modelos. Sirva de ejemplo WorldCat, el principal producto del consorcio, que es un referente en cuanto al volumen de bibliotecas que han apostado por incluir sus fondos en este catálogo colectivo.

Esta etapa que se inicia con la aparición de OCLC y MARC está marcada por la proliferación y generalización de los microordenadores (Rayward, 2002, p. 13), que desplazaron a las grandes computadoras que hasta el momento centralizaban los procesos. Los ordenadores personales han llevado aparejado el nacimiento de los sistemas integrados de gestión de bibliotecas (SIGB). Es en esta etapa cuando se consolidan los modelos comerciales frente a soluciones monofuncionales de otras épocas que habían sido desarrolladas por las propias bibliotecas o por los centros de cálculo de las instituciones (Saorín, 2002, p. 81). La característica principal de los primeros SIGB es que se siguen basando en el traslado de los servicios manuales a la informática, aunque, cada vez más, se producen evoluciones e incorporan funcionalidades que eran impensables en los sistemas monofuncionales.

La aparición de Internet ha supuesto un tremendo cambio para las bibliotecas y centros de documentación y ha provocado el surgimiento de una serie de exigencias que van más allá de la simple emulación de los procedimientos bibliotecarios manuales existentes: La visualización directa de los contenidos digitales, la gestión de accesos a las publicaciones periódicas, o la inclusión de herramientas de descubrimiento son solo algunos ejemplos de requerimientos que no proceden de una actividad manual.
Finalmente, la socialización de Internet ha sido un proceso que comenzó en la década de los 90 con el surgimiento de alternativas al software comercial a partir de otros modelos, conocidos como software libre y de código abierto, pero que ha tenido su máxima explosión a partir de 2000. Y al mismo tiempo, la imparable evolución tecnológica, ha hecho nacer una serie de servicios en la nube que también se conocen como cloud computing.

El cloud computing es un modelo que se basa en la oferta de servicios desde la Web (López Hurtado, Valentín Ruiz y Olivera Zaldua, 2012, p. 334) —de modo que es posible ejecutar desde Internet aplicaciones sin necesidad de tenerlas instaladas en el ordenador o servidor local (Valentín Ruiz, Fernández Sánchez y TorreMarín, 2012, p. 3) - y que ha aparecido como alternativa al software comercial y al software libre y de código abierto. Según Breeding (2012, p. 13) estos servicios podrían sustituir a los SIGB tradicionales en unos pocos años; y se trata de una tecnología en pleno desarrollo, ya que, aunque los servicios en la nube no son novedosos, su aplicación a las bibliotecas, y centros de información no ha sido posible hasta fechas muy recientes, quizá por la complejidad de los programas de gestión.

Por supuesto, hemos de decir que estas etapas que hemos definido no son comunes a todos los centros: Existen instituciones que directamente han pasado de no estar automatizadas a adquirir una licencia SIGB comercial o a contratar un servicio en la nube. En España este estado de no automatización es más que evidente en un volumen importante de centros públicos; ya que, tal y como se extrae de la encuesta elaborada por el Ministerio de Cultura en 2009, en torno al $40 \%$ de las bibliotecas de la Administración General del Estado aún no están automatizadas (Resultados..., 2009, p. 3).

\section{Cloud computing: ventajas e inconvenientes}

Analizada esta evolución de la automatización, hemos de preguntarnos por las razones por las que se están popularizando los servicios cloud computing en bibliotecas, centros de documentación, archivos, etc. Una de ellas está relacionada con la dificultad que tienen los SIGB de incorporar servicios que ya no resultan directamente de la emulación de funcionalidades manuales. Otra se debe a que estas aplicaciones no estaban pensadas en sus orígenes para los, cada vez más comunes, contenidos digitales (Breeding, 2011, p. 26), sino que se centraban en los materiales impresos. Hasta ahora las 
bibliotecas (sobre todo las universitarias) habían resuelto (en parte) la gestión de los contenidos digitales creando repositorios institucionales, también en una clara apuesta por el acceso abierto (Anglada, 2012, p. 554). Por su parte, el acceso al texto completo de los artículos de las publicaciones periódicas se estaba administrando a través de herramientas de resolución de enlaces. Pero los usuarios solicitan un acceso integrado a todos los recursos de la biblioteca; y las herramientas que permitan la agrupación de todos los contenidos se hacen imprescindibles. Son estas las herramientas de descubrimiento, que van más allá de la mera resolución de enlaces.

Del mismo modo, la incorporación de herramientas de la web 2.0 no siempre es sencilla en los tradicionales sistemas de gestión bibliotecaria. Lo mismo ocurre con la adaptación de los SIGB a su visualización y consulta a través de dispositivos móviles. Estos dos son requisitos relacionados con la necesidad de que las bibliotecas y centros de documentación presten sus servicios donde se encuentren sus usuarios (Grande González, Fuente Redondo, 2012, p. 583).

Especial mención requiere el caso de la gestión de los libros electrónicos con restricciones de uso asociadas mediante DRM. Es aquí donde las plataformas cloud computing juegan un papel más destacado al ser el único medio que permite administrar el proceso de circulación de estos contenidos de forma satisfactoria.

Por otro lado, en cuanto a la reducción de costes, debemos plantearnos si es una de las características que definen el modelo. Creemos que un servicio en la nube no tiene porqué incluir necesariamente una reducción de costes aunque lo que sí es cierto es que permite simplificar la gestión de licencias, desarrollos, instalación de actualizaciones, servicios de hosting o almacenamiento, soporte técnico, etc., que tenían las instituciones que adquirían SIGB, porque el servicio cloud computing se contrata incluyendo todos los costes asociados.

En base a estas características que pueden entenderse como necesidades de las bibliotecas y centros de documentación del siglo XXI, podemos extraer una serie de ventajas e inconvenientes que son inherentes al cloud computing.

Entre las ventajas encontramos la mayor facilidad para integrar funcionalidades que no derivan directamente de las tareas manuales desempeñadas tradicionalmente en las bibliotecas y la integración de todos los recursos en un único acceso. Permite agrupar los costes que anteriormente estaban asociados al funciona- miento de los SIGB y que ahora se prestan de manera unitaria a través de una única contratación de servicio. Otra de las ventajas es la autonomía de los usuarios, ya que se trata de herramientas sencillas que permiten la autogestión (López Hurtado, Valentín Ruiz y Olivera Zaldua, 2012, p. 335). La integración automática de herramientas de la web social, la visualización de las plataformas en dispositivos móviles y la posibilidad de contratar estos servicios de forma consorciada son algunas de las características que también definen a los modelos en la nube.

Los inconvenientes están encabezados por la seguridad de los datos. Según afirma Joyanes Aguilar (2012, p. 96) el usuario desconoce las condiciones de almacenamiento de los datos y debe confiar a terceros un aspecto que antes era responsabilidad propia.

\begin{tabular}{ll}
\hline Ventajas & Inconvenientes \\
\hline $\begin{array}{l}\text { Incorporación de } \\
\text { funcionalidades } \\
\text { derivadas }\end{array}$ & Seguridad de los datos \\
\hline $\begin{array}{l}\text { Integración de } \\
\text { contenidos en una } \\
\text { única plataforma }\end{array}$ & $\begin{array}{l}\text { Dificultades para el } \\
\text { desarrollo de nuevas } \\
\text { funcionalidades }\end{array}$ \\
\hline $\begin{array}{l}\text { Herramientas de } \\
\text { descubrimiento }\end{array}$ & $\begin{array}{l}\text { Propiedad del } \\
\text { conocimiento generado }\end{array}$ \\
\hline $\begin{array}{l}\text { Agrupación de costes y, } \\
\text { en ocasiones ahorro }\end{array}$ & $\begin{array}{l}\text { Recuperación de la } \\
\text { información }\end{array}$ \\
\hline $\begin{array}{l}\text { Incorporación de } \\
\text { aplicaciones de la } \\
\text { Web 2.0 }\end{array}$ & $\begin{array}{l}\text { Mayor dependencia de } \\
\text { terceros }\end{array}$ \\
\hline Tecnología móvil & $\begin{array}{l}\text { Corrección de errores e } \\
\text { incidencias }\end{array}$ \\
\hline $\begin{array}{l}\text { Posibilidades de } \\
\text { contratación } \\
\text { consorciada }\end{array}$ & Preservación digital \\
\hline
\end{tabular}

Tabla I. Ventajas e inconvenientes del cloud computing aplicado a bibliotecas y centros de documentación

El desarrollo de nuevas funcionalidades no siempre está permitido en estos servicios, aunque la tendencia sea la de abrir las plataformas en la nube al desarrollo por socios o partners e incluso por los usuarios finales (Valentín Ruiz, Fernández Sánchez y Torre-Marín, 2012, p. 7). La propiedad del conocimiento generado, un asunto que generalmente debe quedar claro en los contratos de servicios, a veces se difumina en los servicios en la nube, sobre todo si dejan de pagarse licencias (Lara Navarra; Maniega Legarda, 2011). Este hecho se relaciona con las posibilidades de recuperación de la información 
pues no en todos los casos las plataformas, cloud computing permiten a los usuarios la descarga del contenido generado.

Finalmente, aspectos como la mayor dependencia de las bibliotecas y centros de documentación de las entidades que proporcionan los servicios en la nube, las limitaciones que imponen algunos proveedores a la hora de proporcionar servicios de soporte y de corrección de incidencias y los temas relacionados con la preservación digital, son aspectos que deberán resolverse para que estos servicios sean definitivamente incorporados.

\section{Bibliotecas y centros de documentación en la nube}

La llegada de los servicios en la nube a las bibliotecas y centros de documentación es pues un camino que tiene por objeto solventar una serie de necesidades que estas instituciones deben satisfacer y que otros modelos no permiten incorporar o lo hacen con mayores dificultades.

Nuestro objetivo en este apartado es el de mostrar ejemplos que permitan ilustrar el funcionamiento de estos servicios y cómo atienden a las características del apartado anterior y, al mismo tiempo, presentar cómo hacen frente a los inconvenientes descritos. En este sentido hemos seleccionado dos plataformas desarrolladas por OCLC: WorldShare y CONTENTdm. Estos dos ejemplos no son únicos en el mercado. En el caso del primero, existen otros productos de similares características como Alma de Ex Libris, o Serials Solutions de Intota. En cuanto a gestores de contenidos digitales como CONTENTdm encontramos también herramientas con grandes posibilidades en el ámbito del código abierto. Es este el caso de DSpace. Sin embargo no es objeto de este trabajo hacer una comparación de diferentes sistemas, sino simplemente mostrar, a modo de ejemplo, unas soluciones concretas a los problemas planteados.

Por otra parte, la elección de WorldShare y CONTENTdm como ejemplos responde a una motivación que tiene mucho que ver con los apartados previos de este trabajo. Cuando hemos descrito la evolución de la automatización de bibliotecas hemos aludido a las etapas fijadas por Rayward (2002, p. 13), que marcaba el inicio de las actividades de OCLC como un hito en esta historia (etapa que hemos denominado como «antes y después de OCLC»). Esto es así porque con esta institución se inician los proyectos de colaboración entre centros de documentación y bibliotecas, y porque ha estado a la cabeza en el desarrollo de tecnología bibliotecaria. De este modo, por la importancia de la institución, hemos seleccionado estos dos servicios para arrojar luz sobre la modalidad a la que nos venimos refiriendo: la tecnología cloud computing.

\subsection{WorldShare en la biblioteca}

WorldShare Management System es un claro ejemplo de lo que Breeding (2012, p. 13) define como "plataformas de servicios bibliotecarios»: una evolución de los tradicionales SIGB. WorldShare aúna todos los módulos de un SIGB: gestión de adquisiciones, catalogación, control de autoridades (integrado con VIAF, Fichero de Autoridades Virtual Internacional), circulación, publicaciones periódicas, préstamo interbibliotecario y catálogo (cuya consulta se realiza a través de WorldCat).

Veamos si atiende a cada una de las ventajas que hemos definido. En primer lugar, permite la gestión de contenidos tanto de soporte tradicional en papel como de todo tipo de material en soporte digital, así como la consulta directa del texto completo de los artículos de las publicaciones periódicas que la biblioteca tenga suscritas, todo a través de una única plataforma y mediante un único acceso para el usuario. El catálogo, WorldCat, ya incorpora una herramienta de descubrimiento y funcionalidades de la web social y de consulta a través de dispositivos móviles. En cuanto a la forma de pago hemos de indicar que Worldshare se contrata mediante una suscripción anual, que se puede realizar de forma consorciada por varios miembros de una red.

WorldShare Management System, como hemos comentado anteriormente, dispone de varios módulos de gestión en la aplicación, siendo muy interesante -por el uso incipiente que están haciendo las bibliotecas hoy en día- la gestión de recursos electrónicos mediante el uso de la denominada WorldCat Knowledge Base, así como la gestión de licencias y proveedores de estos recursos electrónicos, o la posibilidad de integrar un proxy como EZproxy de OCLC. Todo esto se encuentra orientado a que haya una gestión integral de los recursos electrónicos en esta plataforma. Por supuesto, el módulo de adquisiciones está relacionado con el de gestión de recursos electrónicos y licencias, por lo que es posible la adquisición de las colecciones de recursos electrónicos de los diferentes proveedores suscritos.

Por el otro lado, ha sido diseñado para solventar algunos de los inconvenientes definidos. En primer lugar, ofrece la posibilidad de que desa- 
rrolladores externos participen en el diseño de funcionalidades adicionales, mediante el uso de API's. Además, el usuario puede recuperar la información volcada en el sistema en formato MARC21 y las bases de datos de usuarios para cargarlas en otro sistema si decide abandonar el servicio. Los recursos electrónicos suscritos también pueden descargarse en formato MARC21.

Cabe destacar además que esta plataforma se actualiza periódicamente. Normalmente, hay hasta cuatro actualizaciones al año para incluir funcionalidades o corregir problemas detectados. Estas actualizaciones aportan un beneficio constante y recurrente a la comunidad de usuarios, y además demuestran que se trata de una plataforma viva y en constante evolución.

Es interesante destacar que todo el uso, gestión y mantenimiento de la aplicación se realiza a través del navegador web.

El primer caso documentado de aplicación de WorldShare lo encontramos en la Universidad Pepperdine, en Estados Unidos (Dula, 2012) con la que OCLC puso en marcha el servicio en 2012 y que describe el esfuerzo de implantación que sirvió después para las aplicaciones posteriores.



Figura 1. Ejemplo del acceso único al catálogo WorldCat que proporciona WorldShare a la Universidad de Delaware

El interés por el servicio se ha ido incrementando hasta alcanzar las 100 instalaciones en producción el pasado marzo de 2013, cuando la Universidad de Nebraska acometió la migración al sistema (University of Nebraska, 2013). Muchas universidades han apostado por este servicio, como la Universidad de Delaware o la Universidad Tilburg. En España uno de los primeros ejemplos de aplicación es el del Instituto de Empresa, que lo ha incorporado para la gestión de sus bibliotecas en 2012 (IE Library serving, 2012).

\subsection{CONTENTdm}

y la gestión de objetos digitales

CONTENTdm es un gestor de contenidos digitales de todo tipo que permite la administración de colecciones de objetos y de sus metadatos asociados, e incorpora de forma paralela una funcionalidad para la difusión de estos contenidos. Principalmente está pensado para la imagen, pero es posible incorporar de forma satisfactoria documentos audiovisuales y textuales, entre otros.

Pero, analicemos si cumple las características marcadas como ventajas y hasta qué punto es capaz de dar respuesta a los inconvenientes.

Por un lado incorpora funcionalidades que van más allá de lo que es una mera base de datos utilizada para la gestión de imágenes como la exportación de ficheros $\mathrm{xml}$ de metadatos en formato Dublin Core o la posibilidad de crear repositorios OAI-PMH que permitan a recolectores recoger descripciones de forma automatizada. Por defecto, CONTENTdm ofrece una herramienta de descubrimiento que posibilita al usuario la aplicación de filtros personalizados partiendo de unas opciones de búsqueda más o menos complejas, funcionalidad también conocida como facetas.

Integra funcionalidades de la Web 2.0, mediante la integración de la herramienta AddThis (2013) para compartir los objetos digitales en las múltiples plataformas sociales disponibles, así como mediante el uso de contenidos generados por el usuario, tales como comentarios, valoraciones o establecimiento de etiquetas.

CONTENTdm también dispone de un módulo de gestión de usuarios, asignando permisos a determinadas funciones, roles, etc. De esta forma, es fácilmente controlable quién puede acceder y realizar las tareas en la aplicación.

La utilización de este recurso de forma consorciada es posible; pues, por sus características, permite la generación de diferentes sitios web individuales correspondientes a las colecciones de objetos digitales de cada una de las instituciones miembros del consorcio.

Los inconvenientes del cloud computing relativos a la preservación digital se solventan en parte porque CONTENTdm puede interactuar con repositorios propios de las instituciones. En este caso el almacenamiento de los contenidos corre bajo la responsabilidad de las bibliotecas y centros de documentación que contratan el servicio y depende de su política propia de preservación. Esto también está relacionado con la seguridad de los datos, pues las imágenes se 
encuentran bajo disposición de los centros contratantes del servicio y no en los servidores del proveedor.

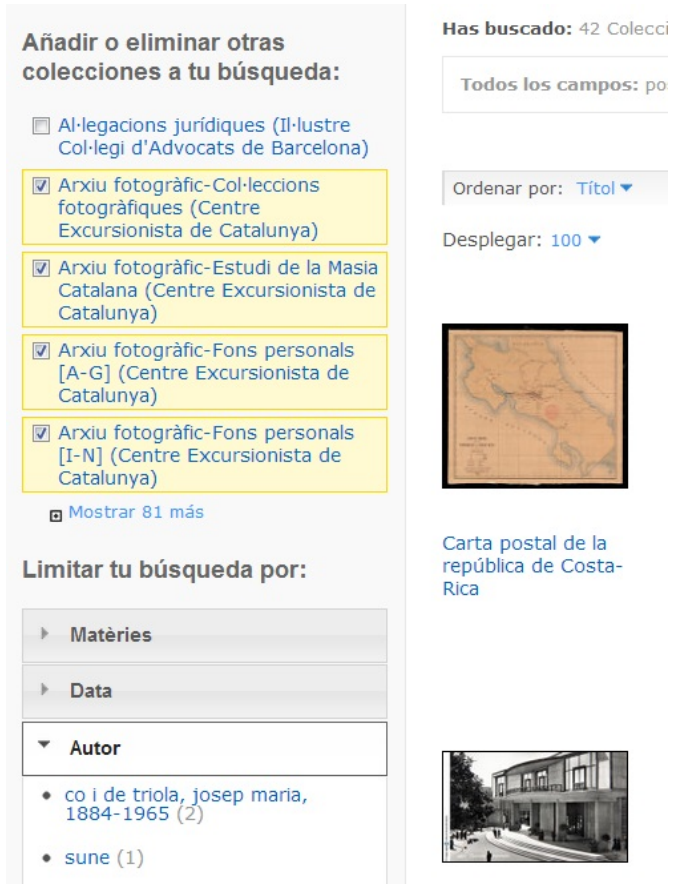

Figura 2. Herramienta de descubrimiento de CONTENTdm: ejemplo de la Memòria Digital de Catalunya

Al igual que WorldShare, CONTENTdm permite la exportación en todo momento de las descripciones de los objetos en formatos estándar como Dublin Core.

Otra de las funcionalidades claves de esta plataforma, es el uso de la WorldCat Digital Collection Gateway (2013). Se trata de una pasarela que permite mapear los registros de la aplicación en formato Dublin Core a formato MARC21, de forma que estos registros acaban siendo trasladados y sincronizados en WorldCat, lo que permite una mayor visibilidad de los fondos digitales que alberga un repositorio de estas características.

Para una mayor facilidad en la descripción de los objetos digitales, en su proceso y subida al servidor, CONTENTdm facilita una herramienta denominada Project Client. Se trata de una aplicación cliente Windows que ofrece un conjunto de herramientas y procesos para la edición y asignación de metadatos, y para conseguir una mayor flexibilidad en la subida de los objetos digitales al servidor web.

En cuanto a los ejemplos de aplicación son varias las instituciones que han apostado por esta plataforma para la gestión de sus conteni- dos digitales como el Consorcio de Biblioteques Universitàries de Catalunya, el Institut Cartogràfic de Catalunya (Roset, Reollo Tudó y TorreMarín, 2012), o el Instituto Cervantes (Iglesia Sánchez, Uguina Corcostegüe y Fuertes Conde, 2011).

También son destacables los acuerdos de colaboración que OCLC ha suscrito con diversas universidades para el uso con fines docentes de CONTENTdm, entre las que destaca la Universidad Carlos III de Madrid, la Universitat de Barcelona y la Universidad Complutense de Madrid, que ha publicado un artículo sobre la experiencia (López Hurtado, Valentín Ruiz y Olivera Zaldua, 2012).

\section{Conclusiones}

Podemos concluir que las plataformas en la nube son una evolución motivada por determinadas necesidades no solventadas de forma satisfactoria por los sistemas integrados de gestión de bibliotecas (SIGB) convencionales.

Además, las aplicaciones basadas en la tecnología cloud computing aún deben evolucionar para dar respuesta a una serie de inconvenientes relacionados con la seguridad de la información que almacenan, las dificultades para el desarrollo de nuevas funcionalidades, la necesidad de asegurar la propiedad del conocimiento generado y de posibilitar a los usuarios la recuperación de la información, la reducción de la dependencia de los proveedores, y los temas relacionados con la preservación digital.

\section{Referencias}

AddThis (2013). http://www.addthis.com/ (2013-07-05).

Anglada, L. (2012). Bibliotecas universitarias: cabalgando la tecnología, siguiendo al usuario. // El Profesional de la Información. 21:6, 553-556.

Breeding, M. (2011). Preparing for the Long-Term Digital Future of Libraries. // Computers in libraries. 31:1. 24-26.

Breeding, M. (2012). Tendencias actuales y futuras en tecnologías de la información para unidades de información. // El Profesional de la Información. 21:1, 9-15. http://www.elprofesionaldelainformacion.com/breedingespanol.pdf

DULA, M.; et al. (2012). Implementing a new cloud computing library management service: a symbiotic approach. // Computer in libraries, 32:1.

García Camarero, E.; García Melero, L. A. (1999). La biblioteca digital. Madrid: Arco, 1999.

Grande González, P.; Fuente Redondo, P. (2012). Bibliotecas universitarias en la web social. // El Profesional de la Información. 21:6, 577-584.

IE Library serving IE Business School in Madrid and IE University in Segovia, Spain, selects OCLC WorldShare Management Services (2012). Dublin (Ohio): OCLC, 27 de septiembre de 2012. http://www.oclc.org/news/ releases/2012/201262.en.html 
Iglesia-Sánchez, Y.; Uguina Cocostegüe, P.; Fuertes Conde, J. M. (2011). Adaptación del gestor de colecciones digitales CONTENTdm en un entorno corporativo. // El Profesional de la Información. 20:6, 689-693. http://eprints.rclis.org/16305/1/689-693\%20(1).pdf

Joyanes Aguilar, L. (2012). Computación en la nube. // Revista del Instituto Español de Estudios Estratégicos. 2012:0, 87-110. http://www.ieee.es/Galerias/fichero/Re vista_Digital/RevistalEEE_Num_0.pdf

Lara Navarra, P.; Maniega Legarda, D. (2011). Conocimiento en la nube: evolución de las intranets. // El Profesional de la Información. 20:2, 171-181.

López Hurtado, M.; Valentín Ruiz; F. J.; Olivera Zaldua, M. (2012). Gestión de Contenidos digitales: Primera aplicación docente de CONTENTdm en la Universidad española: estudios de Grado y Máster en la Facultad de Ciencias de la Documentación de la UCM. // Revista General de Información y Documentación. 22, 333-347.

OCLC (2012). Heritage. http://www.oclc.org/about/history/ default.htm

Rayward, W. B. (2002). A history of computer applications in libraries: prolegomena. // IEEE Annals of the History of Computing. 24:2, 4-15.

Resultados de la encuesta sobre el estado de la automatización de las bibliotecas de la Administración General del Estado (2009). Madrid: Subdirección General de Coordinación Bibliotecaria del Ministerio de Cultura, 2009.

http://www.mcu.es/bibliotecas/docs/MC/BAGE/resultado s_encuesta_BAGEs.pdf

Reynolds, D. J. (1993). Library automation. // Wedgeworth, R. (ed.). (1993). World Encyclopedia of Library and Information Services. Chicago: American Library Association, 1993. 470-476.
Roset, R.; Reoyo Tudó, S.; Torre-Marín, R. (2012). Gestión de objetos digitales con CONTENTdm: estudio de caso. // El Profesional de la Información. 21:1, 91-97. http://www.recercat.net/bitstream/handle/2072/179545/0 91-097-Roset-Reoyo-Torre-1.pdf?sequence=1

Salmon, S. R. (1975). Library automation. // Kent, A.; Lancour, H. y Daily, J. E. (eds.) (1975). Encyclopedia of library and information science. New York: Marcel Dekker. $14,338-345$.

Saorín, T. (2002). Modelo conceptual para la automatización de bibliotecas en el contexto digital. [Tesis doctoral]. Murcia: Universidad de Murcia, 2002.

University of Nebraska Omaha is 100th OCLC WorldShare Management Services user in production (2013). // Library Technology Guides, 6 de marzo de 2013. http://www.librarytechnology.org/ltgdisplaytext.pl?RC=17726.

Valentín Ruiz, F. J.; Fernández Sánchez, A. B.; Torre-Marín, R. (2012). «Servicios cloud computing para la gestión de bibliotecas: hacia un nuevo modelo». // VI Congreso Nacional de Bibliotecas Públicas / Europeana Conference. Burgos: Ministerio de Educación Cultura y Deporte, 9-11 de octubre de 2012. http://www.mcu.es/bibliote cas/docs/MC/2012/CongresoBP/Comunicaciones/Servici oscloudcomputing.pdf.

WorldCat Digital Collection Gateway. (2013). http://www. oclc.org/es-americalatina/digital-gateway.html (2013-0705)

Enviado: 2012-04-04. Segunda versión: 2013-08-07. Aceptado: 2013-08-24. 
